\title{
Pre-evaluación DIT de la resistencia frente a la succión del viento de kits de revestimiento de fachada ventilada con bandejas de paneles composite de aluminio
}

\section{Pre-assessment of the wind-suction resistance of ventilated façades cladding kits based on cassettes made of aluminium composite panel}

\author{
E. Lahoz Ruiz $^{(*)}$, T. Amat Rueda ${ }^{(*)}$, F. Hernández de Olivares ${ }^{(* *)}$
}

\section{RESUMEN}

La pre-evaluación DIT de sistemas constructivos innovadores facilitará la posterior emisión de un DIT mediante una evaluación preliminar pero fiable de aquellas prestaciones críticas relativas a su idoneidad de empleo. Para los kits de revestimiento de fachada ventilada con bandejas horizontales procedentes de PCA rigidizadas por su trasdós, una de éstas es la resistencia frente al viento. Pero no hay un método específico. Se analizó su seguridad desde los ámbitos del aseguramiento frente al riesgo meteorológico y de la normativa. Se estableció como hipótesis que resistirían sin roturas ni deformaciones permanentes significativas, valores de succión de viento de hasta $96 \mathrm{~km} / \mathrm{h}$. Se ensayaron diferentes bandejas en una maqueta a escala real. Se concluye que estos kits serán seguros si resisten un valor de 2,4 kPa sin rotura, y se limitan las deformaciones acumuladas instantáneas y residuales a valores máximos respectivos de L/30 y L/300, siendo L la distancia entre apoyos.

Palabras clave: Succión; kits de fachadas ventiladas; bandejas; paneles composite de aluminio.

\section{ABSTRACT}

The pre-assessment DIT of innovative building kits will facilitate the posterior issuing of a Technical Agrément throughout a preliminary but reliable assessment of fitness for use based on their critical performances. For cladding kits based on horizontal stiffened cassettes made of ACP, one of them is the resistance to wind loads. But there is no specific method available yet. It has been analysed its safety in the field of assurance against meteorological risks and regulations. It has been established as hypothesis, that kits would resist winds of speed up to $96 \mathrm{~km} / \mathrm{h}$ with no breakage neither significant permanent deflections. Different cassettes were tested on a real-scale rig built up with different cassettes. Results lead to conclude that these kits will be safe against wind suction if they resist 2,4 kPa without breakages, and accumulated instantaneous and permanent deflections are restricted respectively to values up to $L / 30$ and $L / 30 O$, where $L$ is the span between supports.

Keywords: Suction; ventilated cladding kits; cassettes; aluminium composite panels.

(*) Instituto de Ciencias de la Construcción Eduardo Torroja (IETcc-CSIC), Madrid (España).

${ }^{(* *)}$ Escuela Técnica Superior de Arquitectura - Universidad Politécnica de Madrid (España).

Persona de contacto/Corresponding author: elahoz@ietcc.csic.es (E. Lahoz Ruiz)

Cómo citar este artículo/Citation: Lahoz Ruiz, E., Amat Rueda, T., Hernández de Olivares, F. (2015). Pre-evaluación DIT de la resistencia frente a la succión del viento de kits de revestimiento de fachada ventilada con bandejas de paneles composite de aluminio. Informes de la Construcción, 68(542): e143, doi: http://dx.doi.org/10.3989/ic.15.070.

Licencia / License: Salvo indicación contraria, todos los contenidos de la edición electrónica de Informes de la Construcción se distribuyen bajo una licencia de uso y distribución Creative Commons Attribution License (CC BY) Spain 3.o. 


\section{INTRODUCCIÓN}

Los kits de revestimiento de fachada ventilada a base de bandejas colgadas procedentes de paneles composite de aluminio son sistemas constructivos no normalizados que presentan un uso muy extendido en España. Como sistemas innovadores, pueden ser objeto de Documentos de Idoneidad Técnica, documentos emitidos por el IETcc siempre que su completa evaluación técnica de la idoneidad de empleo para el uso previsto resultara favorable. Como panel composite de aluminio (PCA), puede definirse a un tipo de panel sándwich metálico y delgado, de unas dimensiones estándar habituales de $5000 \mathrm{~mm}$ de longitud y $1500 \mathrm{~mm}$ de anchura y $4 \mathrm{~mm}$ de espesor, compuesto de dos láminas de aluminio aleado de $0,5 \mathrm{~mm}$ de espesor adheridas a un núcleo no aislante de $3 \mathrm{~mm}$ de espesor de polietileno de baja densidad, (panel tipo «PE») o bien de compuestos minerales añadidos a una matriz de polietileno de baja densidad (panel tipo «FR»). Estos aplacados se distinguen por su menor rigidez y mayor ligereza (aproximadamente $5,5 \mathrm{~kg}$ / $\mathrm{m}^{2}$ para un espesor de $4 \mathrm{~mm}$ ), versatilidad de formas, tamaños y acabados.

Así, pueden convertirse en bandejas mediante, corte, fresado, plegado y fijación de pletinas en sus esquinas. En su pestaña superior horizontal cuentan con un pliegue complementario mientras que en su pliegue inferior horizontal pueden contar con uno opcional (doble pestaña). Los pliegues verticales tienen una profundidad habitual de entre 35 y $50 \mathrm{~mm}$ y presentan varias entalladuras (muescas troqueladas) en general con forma de «L». Las entalladuras pueden ir reforzadas con pletinas remachadas que, al encajarse sobre las piezas de cuelgue, permiten colgar la bandeja a la subestructura (véase Figura 1). En este caso, el sistema resultante reviste las fachadas con bandejas de modulación vertical, que pueden presentar rigidizadores horizontales adheridos por su trasdós cuyo estudio no es objeto del presente artículo.

Para revestir las fachadas con bandejas de modulación horizontal, se emplean rigidizadores verticales adheridos por su trasdós (véase Figura 2), capaces de:

- Transmitir las cargas de peso propio y viento y limitar la deformación del aplacado, mediante fijaciones de ocultas mecánico-adhesivas capaces de aportar cierta elasticidad a la unión entre aplacado y subestructura.

- Optimizar el aprovechamiento del tamaño de los paneles, minimizando recortes en la conformación de las bandejas, y conseguir un aspecto estético sin fijaciones vistas.

Estos rigidizadores consisten habitualmente en perfiles angulares fabricados a partir del propio PCA, que además se fijan mediante remachado sobre sus extremos y perpendicularmente, a las pestañas horizontales de las bandejas.

El objeto del presente artículo es permitir evaluar de manera rápida, preliminar y fiable, si el comportamiento de este tipo de revestimientos frente a la succión del viento es suficientemente seguro. Se estableció como hipótesis que las bandejas, si se dimensionan, instalan y mantienen adecuadamente, pueden resistir sin rotura ni deformaciones residuales significativas la succión causada por vientos que la jurisprudencia no aprecia como supuestos de fuerza mayor, es decir aquellos cuya velocidad media no sea superior a $96 \mathrm{~km} / \mathrm{h}$.

\section{ESTADO DEL ARTE}

Una nueva línea de investigación, denominada pre-evaluación DIT (1), busca favorecer la innovación en el sector de la industria de productos de construcción, mediante una evaluación preliminar y fiable de su idoneidad de empleo, centrándose en sus prestaciones críticas. Para este tipo de kits, dos casos de desprendimientos coincidentes con el paso de vendavales indican que la resistencia frente a la succión del viento es precisamente, una de estas prestaciones críticas (2).

El comportamiento de una bandeja de panel composite frente a la succión del viento se puede descomponer en la suma de dos comportamientos a flexión, el de su cara vista más el de sus pestañas perimétricas. La cara vista se modela como una placa plana apoyada en todo su perímetro, ya que el fresado y posterior plegado de las pestañas del panel permitiría el giro relativo de la cara vista de la bandeja respecto de todas las pestañas (3). Para dimensionar esta placa plana frente al viento, se ha de considerar su comportamiento elástico frente a esfuerzos de flexión. Por otra parte, tanto las pestañas verticales como los rigidizadores verticales adheridos se modelan como vigas continuas o bien de un solo vano. Esto depende de su número de entalladuras, que se modelan como apoyos fijos, permitiendo el giro relativo de la viga. Estas premisas, de acuerdo con los DIT específicos emitidos hasta la fecha por el IETcc requieren de las siguientes condiciones:

- Limitando la resistencia admisible de tracción de las chapas, esto es, minorando con un coeficiente de seguridad de 1,75 el límite elástico a tracción de la aleación de aluminio considerada (4).

- Limitando la deformación instantánea, en el centro de la bandejas a L/30, siendo L, la distancia máx. horizontal entre pestañas verticales y/o rigidizadores adheridos (5).

Conviene recordar que en una fachada ventilada con junta abierta sometida a presión del viento, las cargas se reparten sobre montantes y travesaños de forma que todos los elementos trabajan en colaboración; mientras que, sometida a succión, corresponde inicial y principalmente a las fijaciones del aplacado a la subestructura, contrarrestar la tracción que ejercerá el viento. En el tipo de kits estudiado, debido a su menor rigidez, resulta además fundamental limitar la deformación del aplacado.

Por otro lado, es sabido que el aire es un fluido que, al interrumpir su movimiento por encontrar un obstáculo como por ejemplo una fachada de un edificio, intercambia la energía cinética en presión. En realidad, debido a la distorsión del flujo que se genera alrededor de un edificio, se producen presiones o succiones que dependen de factores tales como la velocidad del viento, el ángulo de ataque, o la geometría del obstáculo. En el caso de edificios de forma paralelepipédica, en los cambios de planos se producen remolinos que pueden generar valores locales de succión sustancialmente mayores a los de la presión recibida por la fachada situada a barlovento. En consecuencia, la evaluación de la resistencia de un kit de revestimiento de fachada ventilada frente a la acción del viento suele restringirse al menos inicialmente, al estudio de su comportamiento frente a la succión. Así, ya en la Norma UNE 41.957-1. 2000 (6), se considera, en base a la Norma alemana DIN 1055 sección 4, el efecto local de succión en las zonas de esquinas de los planos de fachada paralelos a la dirección del viento. En concreto, se indica que 

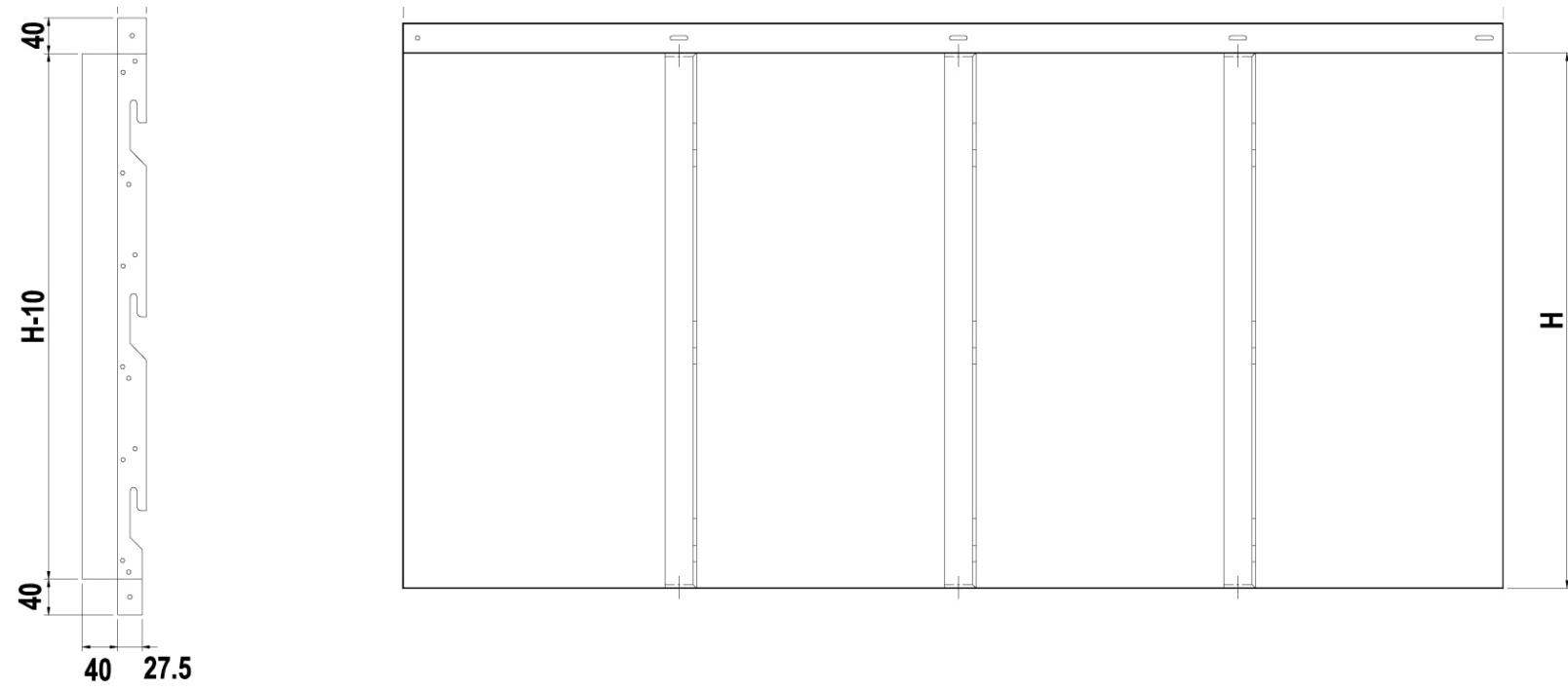

\section{Cordón adhesivo}
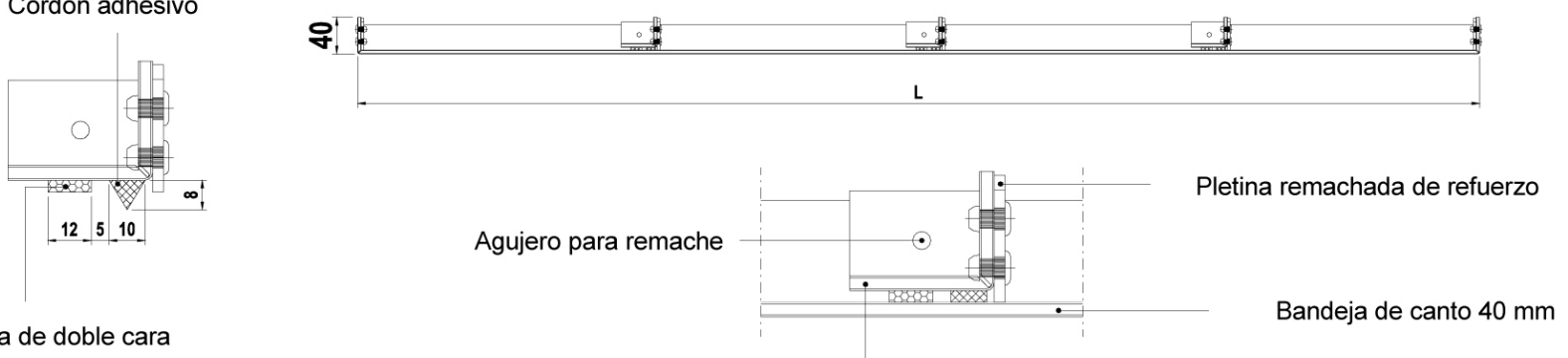

Rigidizador vertical de bandeja de $40 \mathrm{~mm} \mathrm{PCl}$

32.5

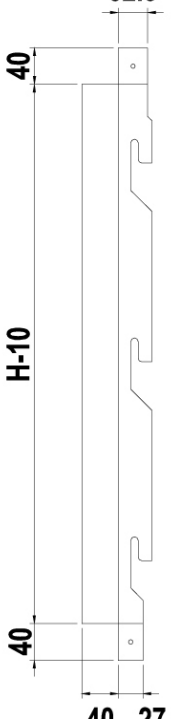

L

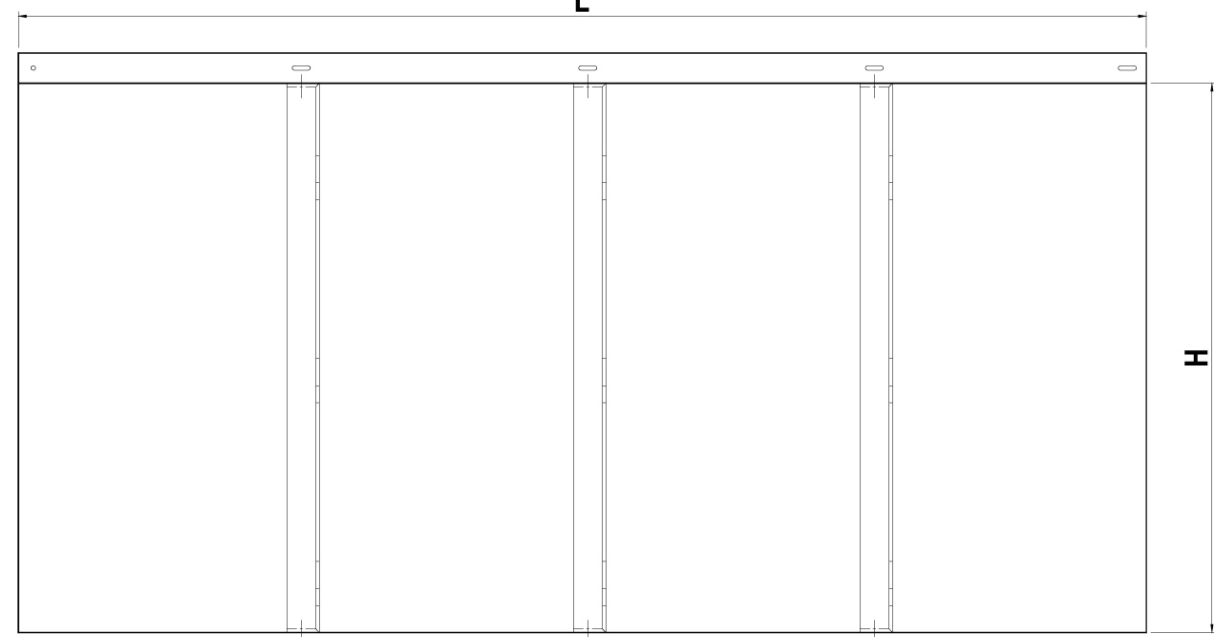

$40 \quad 27.5$

Cordón de adhesivo
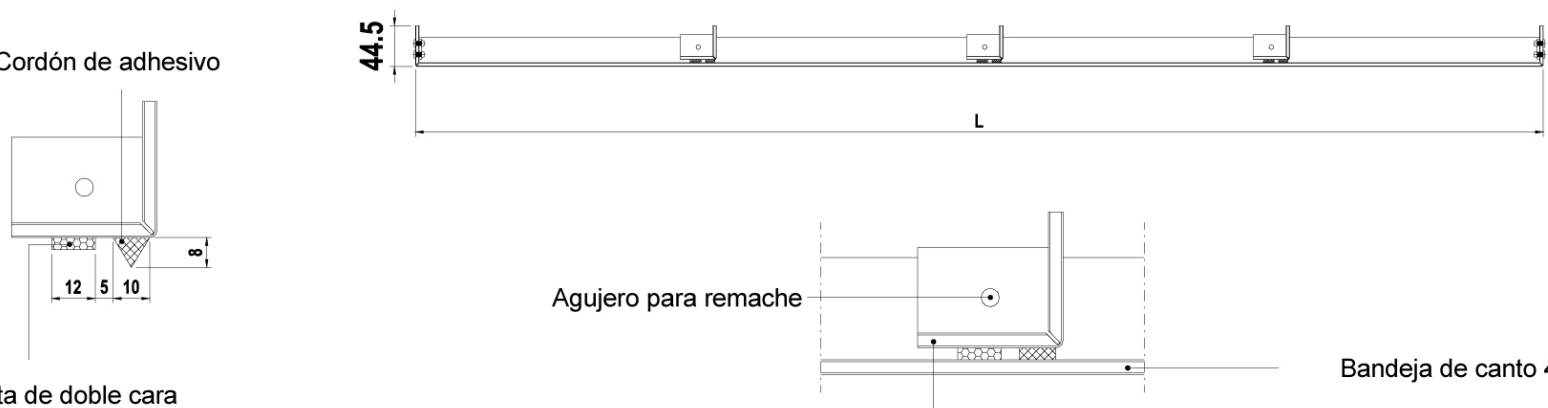

Bandeja de canto $44^{\prime} 5 \mathrm{~mm}$

Cinta de doble cara

Rigidizador vertical de bandeja de $40 \mathrm{~mm} \mathrm{PCl}$

Figura 1. Bandeja de modulación vertical con entalladuras reforzadas con pletinas remachadas. 

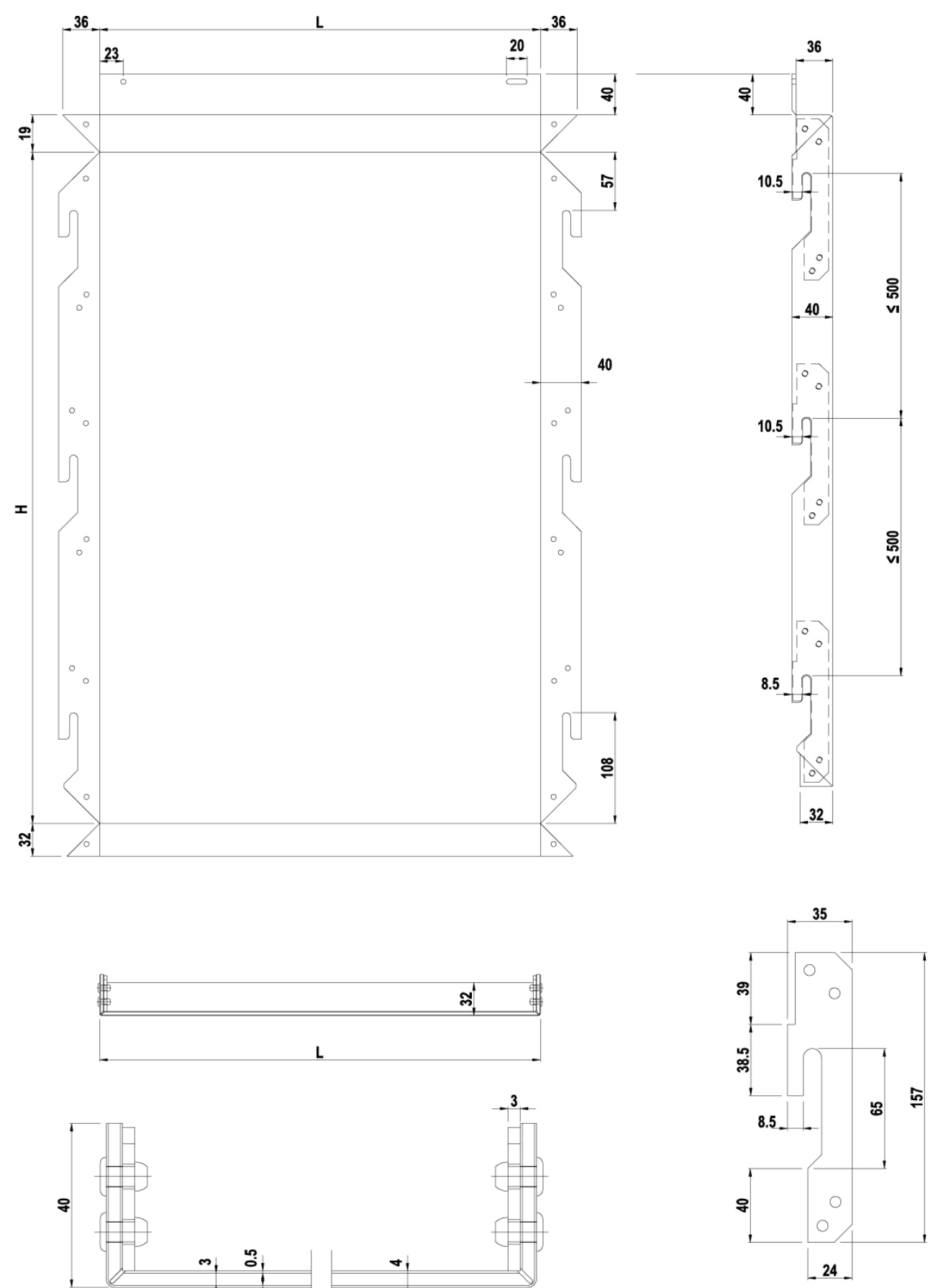

Figura 2. Ejemplo de bandeja de modulación horizontal con rigidizadores adheridos por su trasdós, con y sin entalladuras reforzadas con pletinas remachadas.

el valor de la succión del viento en las esquinas y borde de coronación de las fachadas de un edificio, y a lo largo de una franja de $2 \mathrm{~m}$ de longitud puede ser considerado el orden del doble del valor de la presión sobre la fachada situada a barlovento, (véase Figura 3).

\section{METODOLOGÍA}

A partir de dos casos de desprendimiento de aplacado causados por la acción del viento, se ha estudiado su peligrosidad en los ámbitos de la meteorología, protección civil, y aseguramiento. Se ha revisado la normativa de cálculo aplicable en España. A continuación se han definido las muestras de estudio y se ha calculado la deformación y tensión máxima admisible. Posteriormente se realizaron ensayos de resistencia a la succión del viento.

\subsection{Estudio de antecedentes}

Se investigó en primer lugar la existencia de antecedentes de desprendimientos, como punto de partida de la investigación. Se encontraron los siguientes:

- 28-29 de noviembre de 2005: Caída de bandejas de modulación horizontal y de algunos montantes, en fachadas de la Torre II de Santa Cruz, edificio residencial de $120 \mathrm{~m}$ de 

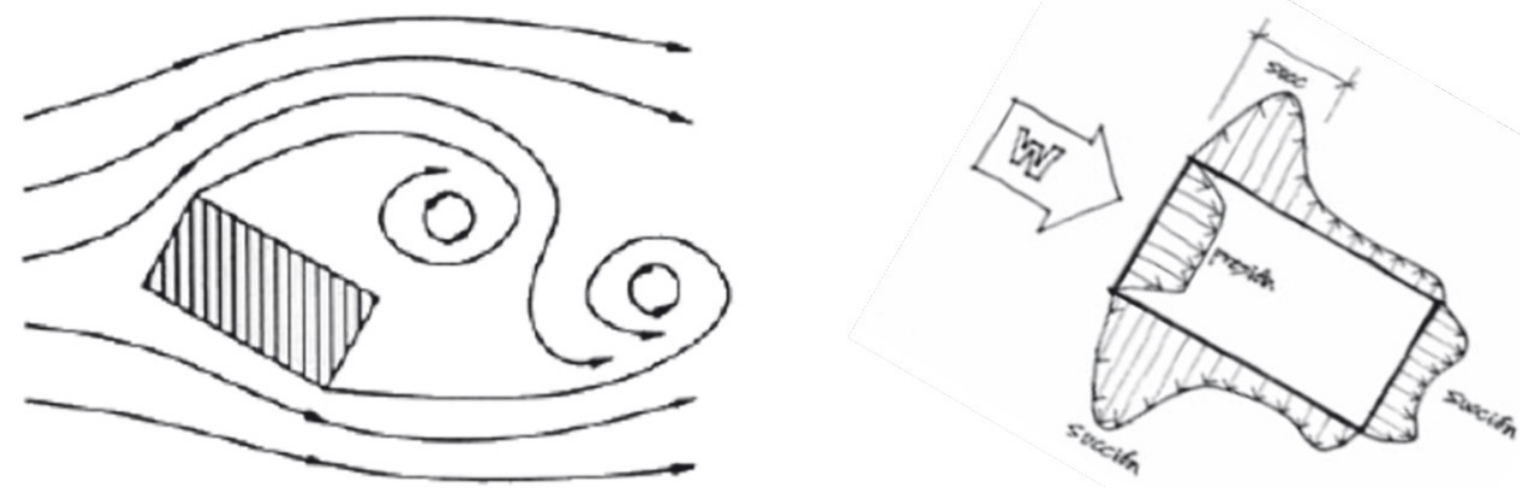

Figura 3. Acción del viento sobre las fachadas de un edificio.

altura sito en C/ Los Llanos Seis, 3. 38003 Santa Cruz de Tenerife, al paso de la tormenta Delta (7).

- 4-6 de febrero de 2014: Desprendimiento de bandejas de modulación horizontal de fachadas Oeste de la sede para la Xunta de Galicia, edificio de $45 \mathrm{~m}$ de altura sito en la Av. Fernández Ladreda, 43. 36001 Pontevedra al paso del ciclón extra tropical Qumaira (8).

Las principales zonas afectadas resultaron ser las esquinas. Esta circunstancia corroboró las indicaciones descritas en el Estado del Arte que suponían a la necesidad de aumentar cuantitativamente la resistencia frente al viento de los elementos de aplacado de las esquinas, por ejemplo reduciendo distancias entre perfiles verticales y/o aumentando la densidad de fijaciones y por supuesto, en la elección adecuada del anclaje del elemento de fijación al soporte.

\subsection{Análisis de la peligrosidad del viento}

Según el Manual de la Agencia Estatal de Meteorología, en adelante AEMET (9), la velocidad del viento se expresaba en función de valores medios (entendidos como promedio de períodos de diez minutos), o bien referenciando a su racha, es decir, la velocidad instantánea y máxima, registrada en el día, expresada en $\mathrm{km} / \mathrm{h}$ y acompañada de la hora de aparición, que supone una desviación transitoria respecto a su valor medio. Así, los vientos se clasifican por AEMET como:

- Calma: Velocidad media menor o igual a $5 \mathrm{~km} / \mathrm{h}$.

- Flojos: Velocidad media entre $6 \mathrm{~km} / \mathrm{h}$ y $20 \mathrm{~km} / \mathrm{h}$.

- Moderados: Velocidad media entre $21 \mathrm{~km} / \mathrm{h}$ y $40 \mathrm{~km} / \mathrm{h}$.

- Fuertes: Velocidad media entre $41 \mathrm{~km} / \mathrm{h}$ y $70 \mathrm{~km} / \mathrm{h}$.

- Muy fuertes: Velocidad media entre $71 \mathrm{~km} / \mathrm{h}$ y $120 \mathrm{~km} / \mathrm{h}$, donde $96 \mathrm{~km} / \mathrm{h}$ sería el valor central (equidistante) entre estos valores extremos.

- Huracanados: Velocidad media mayor que $120 \mathrm{~km} / \mathrm{h}$.

En consecuencia, para no crear confusión, el Manual de AEMET sólo emplea el término racha de viento cuando se superen los $70 \mathrm{~km} / \mathrm{h}$ y entonces se clasifican como:

- Rachas muy fuertes: Cuando superen o hayan superado los $70 \mathrm{~km} / \mathrm{h}$.

- Rachas huracanadas: Cuando superen los 120 km/h.

En el Plan Nacional de predicción y vigilancia de fenómenos meteorológicos adversos (10), en adelante Plan Nacional, las rachas estructuran un rango creciente de niveles de alerta (amarillo, naranja y rojo) y riesgos indicados a continuación, cuantificados mediante unos «valores umbral» de velocidad de racha en km/h, para cada región de España:

- Nivel amarillo: No existe riesgo meteorológico para la población en general aunque sí para alguna actividad concreta (fenómenos meteorológicos habituales pero potencialmente peligrosos) o localización de alta vulnerabilidad, como una gran conurbación.

- Nivel naranja: Existe un riesgo meteorológico importante (fenómenos meteorológicos no habituales y con cierto grado de peligro para las actividades usuales).

- Nivel rojo: El riesgo meteorológico es extremo (fenómenos meteorológicos no habituales, de intensidad excepcional y con un nivel de riesgo para la población muy alto).

Así por ejemplo para los casos referidos se obtendrían los siguientes valores:

- Tenerife (Norte de Tenerife). Nivel amarillo: $\geq 70$. Nivel naranja: $\geq 90$. Nivel rojo: $\geq 130$

- Pontevedra (Rías Baixas): Nivel amarillo: $\geq 80$. Nivel naranja: $\geq 100$. Nivel rojo: $\geq 140$

En el ámbito del aseguramiento de bienes y personas, el Real Decreto 300/2004 (11), después actualizado por el Real Decreto 1386/2011 (12) es dónde se establece el Reglamento del seguro de riesgos extraordinarios. Aquí se regulan las circunstancias donde sería susceptible la intervención del Consorcio de Compensación de Seguros. En este R.D. se define como "acontecimientos extraordinarios", entre otros, a la tempestad ciclónica atípica, que se entiende un tiempo atmosférico extremadamente adverso y riguroso producido, entre otros fenómenos por:

- Ciclones violentos de carácter tropical, identificados por la concurrencia y simultaneidad de vientos con velocidad superior a $96 \mathrm{~km} / \mathrm{h}$ promediados sobre intervalos de $10 \mathrm{mi}$ nutos.

Se reseña la coincidencia con el valor central (96 km/h) del rango de velocidades (71 a $120 \mathrm{~km} / \mathrm{h}$ ) establecida para la categoría de viento muy fuerte según el Manual de AEMET.

- Vientos extraordinarios, definidos como aquellos que presenten rachas que superen los $120 \mathrm{~km}$ por hora, entendiendo por racha el mayor valor de la velocidad del viento, sostenida durante un intervalo de tres segundos. Se reseña que este valor es el establecido para la categoría rachas de viento huracanadas según el Manual de AEMET. 


\subsection{Estudio de la acción del viento en la Normativa: Eurocódigo 1 y Código Técnico (CTE)}

Aunque con ciertas divergencias (13), el Documento Básico de Seguridad Estructural. Acciones en la edificación (en adelante DB SE-AE), de la parte II del Código Técnico de la Edificación, describía los mismos procedimientos y fórmulas para cuantificar la carga de viento que Eurocódigo 1 sobre las acciones del viento (14). Ahora bien, en el DB SEAE no se considera la influencia de los efectos dinámicos, y la estimación de las acciones se determina mediante el cálculo de la presión estática del viento como $q e=q b$. $c e$. cp, donde:

- $q b$ : Presión dinámica, que es función de la zona eólica (A, B y C), y es el parámetro que considera el valor de la velocidad básica del aire. Curiosamente, el valor de $96 \mathrm{~km} / \mathrm{h}$ referenciado en el Real Decreto 300/2004 es inferior al valor máximo indicado en el Anejo D del Código Técnico DB.SE.AE para España (104 km/h en zona C).

- ce: Coeficiente de exposición (en función del grado de aspereza del entorno (de I a V) y la altura del punto considerado.

- cp: Coeficiente de eólico o de presión/succión, que es función entre otras variables de la fachada expuesta (así por ejemplo, la franja «A» sería la más desfavorable)

Así, en determinados casos algunos autores sugirieron para considerar los efectos dinámicos, realizar un estudio ad hoc mediante ensayos en túneles de viento sobre maqueta a escala del edificio en cuestión. El motivo, según Pindado, Francini y Meseguer (15), es que «la presión dinámica de un flujo turbulento sería mayor que la presión dinámica asociada a la velocidad media del aire». En particular, estos autores afirmaban que determinados fenómenos dinámicos, producidos al incidir el viento sobre zonas determinadas de un edificio, podían generar importantes efectos de succión local. Uno de estos fenómenos era la aceleración de la corriente, que puede ser provocada por un encajonamiento del flujo de aire, como cuando dos volúmenes, se encuentran separados pero próximos entre sí.

\subsection{Análisis del procedimiento de ensayo de resistencia a la succión del viento}

En el apartado 5.4.1. de la Guía de la EOTA nº 034 para la concesión del DITE a los sistemas de fachada ventilada emitida en 2012, se describe un procedimiento de ensayo para determinar su resistencia frente a la succión del viento. No obstante, aunque los kits a base de bandejas colgadas con rigidizadores adheridos procedentes de PCA no entraron en su ámbito de aplicación en el momento de su redacción, se considera que puede ser de aplicación este apartado. Este ensayo consiste en someter una maqueta del sistema de revestimiento, en la configuración más desfavorable posible y a escala real, incluyendo la subestructura, frente a la acción simulada de la succión del viento. Es un ensayo dinámico, no estático. Es decir, en él, las cargas se aplican uniformemente repartidas sobre la cara exterior del sistema de manera pulsatoria y creciente, es decir, se aplica de o hasta el valor considerado a una velocidad de $0,2 \mathrm{kPa} / \mathrm{s}$ como máximo. Luego se descarga con idéntica velocidad y se mide la deformación residual o permanente tras un minuto de estabilización, en los puntos considerados. Las cargas se aplican en los siguientes escalones:
- Precarga de dos escalones de 300 Pascales con descarga intermedia.

- Carga de 500 Pascales (o,5 kPa), descarga a cero y carga de 1000 Pascales $(1,0 \mathrm{kPa})$.

- Y a partir de entonces, cargas crecientes en escalones de $200 \mathrm{~Pa}$ en $200 \mathrm{~Pa}$, hasta que ocurra alguna o algunas de las siguientes situaciones:

- Se registre una deformación diferida (permanente) acumulada significativa del aplacado ( $3 \mathrm{~mm}$ según las evaluaciones técnicas europeas emitidas por el IETcc), que suponga irrelevante la continuación del ensayo.

- Suceda la rotura del elemento de aplacado, y/o el fallo de fijaciones, $\mathrm{y} / \mathrm{o}$ bien el desprendimiento de la subestructura.

En cuanto al rango de escalones de succión, en relación con los posibles fallos que se consideran en la Guía DITE 034, se precisa que no se produjeron ni rotura de elementos de aplacado, ni fallos de fijación, ni desprendimientos de componentes de la subestructura, limitando la deformación diferida (permanente) significativa a un máximo de $3 \mathrm{~mm}$, valor considerado visualmente apreciable.

Las dimensiones de las muestras de ensayo, no quedan definidas en la Guía DITE o34, simplemente se indica que al menos se ensaye aquella o aquellas configuraciones que sean más desfavorable. El equipamiento se compone de un bastidor metálico sobre el que se fijan las ménsulas del sistema. Una vez fijados los montantes a las distancias especificadas, se colocan las piezas de aplacado del tamaño considerado con juntas abiertas horizontales y verticales, simulando las configuración real de la obra. A continuación se ha de enfrentar a un cajón abierto de $2400 \mathrm{~mm} \times 2000 \mathrm{~mm}$ de dimensiones interiores de paneles de fibras de madera y media densidad, de modo que perimétricamente se ajuste al bastidor y selle para evitar el paso del aire.

\subsection{Experimentación}

En el contexto de una evaluación rápida, preliminar y fiable de la idoneidad de empleo, se ha de optimizar la fase experimental. Es imprescindible considerar un reducido número de ensayos sobre configuraciones de muestras del sistema que por un lado, se adapten de forma óptima a las dimensiones del cajón de ensayo, sean suficientemente representativas de la puesta en obra y además, según indica la Guía ETAG 034, sean lo más desfavorables posibles. Para este fin, se utilizó una única maqueta de ensayo (véase Figura 4) cuyas características principales eran las siguientes:

- Composición de subestructura:

- Ménsulas: 3 unidades por montante, de menor profundidad posible, (en total 9) fijadas a la perfilería del bastidor metálico mediante tornillos pasantes, arandela y tuerca.

- Montantes: 3 unidades de 2375 mm de longitud, distanciados entre sí $910 \mathrm{~mm}$.

- Piezas de cuelgue: 2 unidades para otras tantas entalladuras por pestaña o rigidizador vertical, fijadas al montante con las fijaciones previstas (p.ej. tornillos).

- Composición del revestimiento: Bandejas horizontales procedentes de PCA de $4 \mathrm{~mm}$ de espesor, rigidizadas verticalmente por su trasdós, compuestas por núcleos tipo «PE» $\mathrm{o}$ «FR» $\mathrm{y}$ por láminas de aleación de aluminio del 

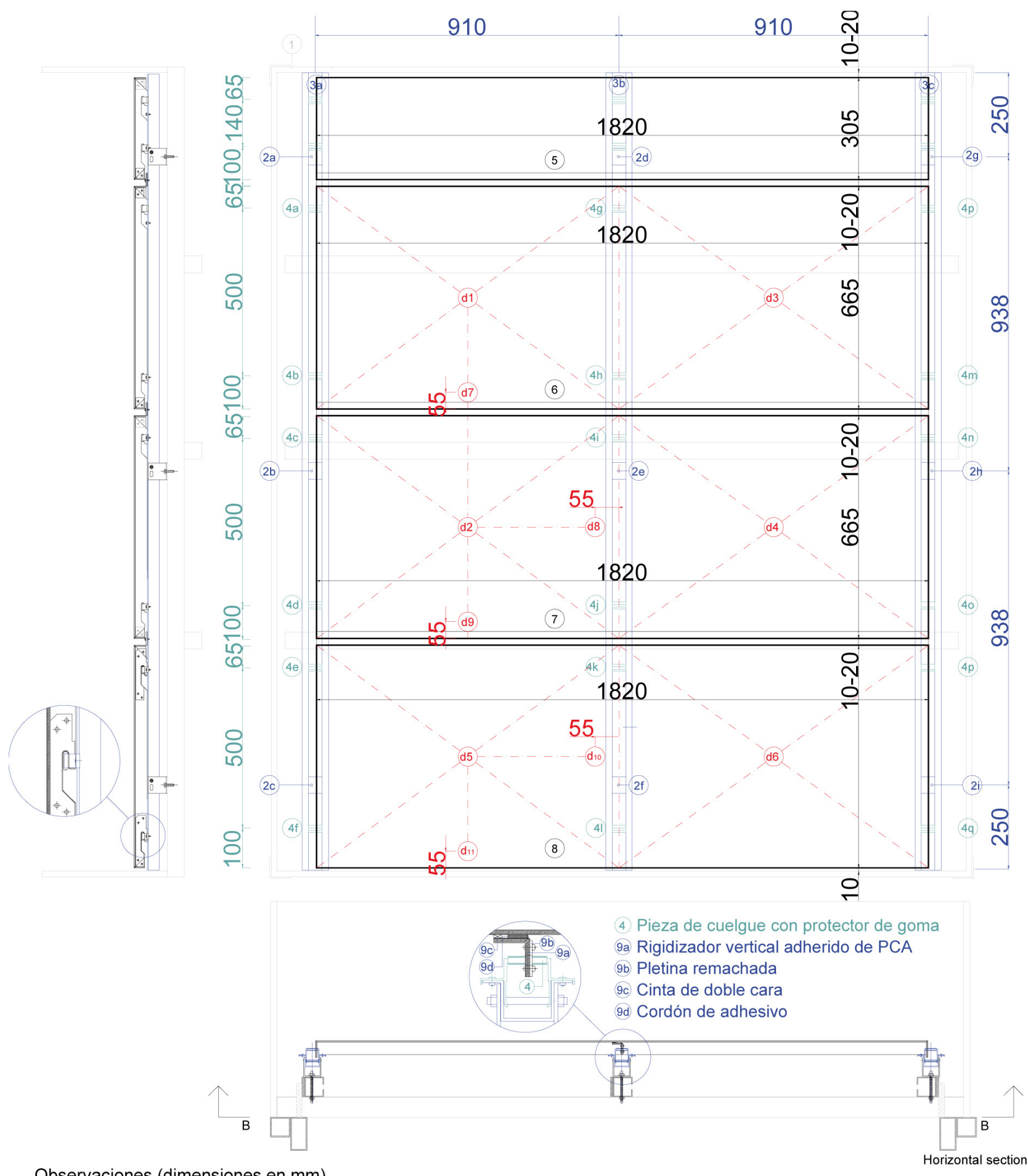

\section{Observaciones (dimensiones en $\mathrm{mm}$ )}

Banco de ensayos compuesto por bastidor y cajón de cierre

2. Ménsula (menor profundidad y canto; max. vano voladizo $=250 \mathrm{~mm}$; máx vano entre ménsulas: $938 \mathrm{~mm})(9$ uds)fijada a bastido।

(3) Montante (longitud=2375 mm) (3 uds) fijado a ménsula

4. Pieza de cuelgue ajustable en altura (24 uds) fijada a montante

(5) Bandeja de cierre ( $\mathrm{LxH}$ cara vista=1820 x $305 \mathrm{~mm}$ ) sin sobrepestaña hor. sup. procedente de panel composite de aluminio PCA

6) Bandeja ( $\mathrm{LxH}$ cara vista=1820 x $665 \mathrm{~mm}$ ) pest.lateral 1 vano prof. $44.5 \mathrm{~mm}$, anch. entalladura no ref. $15 \mathrm{~mm}$, procedente de PC/ tipo PE de $4 \mathrm{~mm}$ de espesor (1 ud)

(7) Bandeja ( $\mathrm{LxH}$ cara vista=1820 x $665 \mathrm{~mm}$ ) pest.lateral 1 vano prof. $44.5 \mathrm{~mm}$, anch. entalladura no ref. $15 \mathrm{~mm}$, procedente de PC/ tipo FR de $4 \mathrm{~mm}$ de espesor (1 ud)

(8) Bandeja ( $\mathrm{LxH}$ cara vista $=1820 \times 665 \mathrm{~mm}$ ) pest.lateral 1 vano prof. $40 \mathrm{~mm}$, anch. entalladura reforz. $10 \mathrm{~mm}$, procedente de PCA tipo FR de $4 \mathrm{~mm}$ de espesor (1 ud)

(d) Medidor de deformación

Figura 4. Esquema de composición de maqueta para ensayo de succión con tres bandejas. 
tipo 5005. La profundidad de sus pestañas verticales era de $44 \mathrm{~mm}$, o bien de $40 \mathrm{~mm}$, y la anchura de lengüeta de las entalladuras: $15 \mathrm{~mm}$, o bien de $10,5 \mathrm{~mm}$, en este caso con entalladuras reforzadas a base de pletinas remachadas. Estos rigidizadores no presentaban más de un vano, por lo que no podían modelarse como vigas continua.

En la definición de las maquetas para ensayo se consideraron las dimensiones interiores del cajón, $2000 \mathrm{~mm}$ de longitud $x$ $2400 \mathrm{~mm}$ de alto, y la estructura del bastidor-soporte, para permitir la ubicación de medidores de deformación LVDT, así como para el ensayo conjunto de tres bandejas de modulaciones horizontal con rigidizadores adheridos. Tal y como se indica en la Tabla 2 y en la Figura 4, los medidores de deformación LVDT se instalaron en los puntos donde a priori se podrían obtener las deformaciones más desfavorables.

Por otra parte, para la posterior discusión de los resultados de ensayo se ha correlacionado tal y como indica en la Tabla 1, los casos de partida considerados, los valores de velocidades del viento para la clase viento muy fuerte establecida en el Manual de AEMET, el límite del equipo de ensayo, y los parámetros del marco normativo (presión dinámica $q b$, coeficiente de exposición ce (edificio de altura $\geq 30 \mathrm{~m}$ y grado I), coeficiente eólico $c p$, (franja A de fachada del edificio) con el escalón de succión del procedimiento de ensayo establecido en la Guía ETAG 034.

\subsection{Cálculos}

\subsubsection{Cálculo del espesor equivalente}

Para predimensionar las bandejas se ha considerado que la cara vista de la bandeja es una placa plana sólida de un espesor «equivalente» al del PCA, cuyas chapas serían de idéntica aleación que las láminas de PCA, caracterizadas por los siguientes datos:

- Tensión máxima admisible de la lámina de aluminio, considerada como el limite elástico del aluminio, minorado con un coeficiente de seguridad, declarado por el fabricante $(1,75)$, lo que supone $90 \mathrm{MPa} / 1,75=51 \mathrm{MPa}$.
- Módulo de Young (E) del material de la lámina (aluminio) $=70.000 \mathrm{MPa}$.

Para calcular el espesor «equivalente» ha sido necesario determinar en primer lugar la rigidez a flexión de una placa sólida equivalente a la rigidez del panel composite.

Para su determinación se partió de igualar la rigidez a flexión de PCA con la rigidez a flexión de la placa sólida y después se procedió a despejar la variable espesor. Se consideró como punto de partida que el comportamiento a flexión de un PCA es como el de un panel sándwich, pero modificado a causa de su delgado espesor. El comportamiento mecánico de un panel sándwich frente a esfuerzos de flexión es hoy bien conocido gracias a Howard G. Allen (16) y Plantema (17). Es frecuente encontrarlos citados en la bibliografía específica. Por ejemplo Zenkert (18), resume el cálculo de la rigidez a flexión D de un panel sándwich de acuerdo con la ecuación siguiente [1]:

$$
\begin{gathered}
\mathrm{D}=\int \mathrm{E} \cdot \mathrm{z} 2 \mathrm{dz}=(\text { Ef.tf3 } / 6)+(\text { Ef.tf. d2 /2) }+(\text { Ectc3/12 })= \\
=2 \mathrm{Df}+\mathrm{Do}+\mathrm{Dc}
\end{gathered}
$$

donde, según la Figura 5:

- Df: Rigidez a flexión de la lámina respecto de su línea neutra individual:

$$
\text { Df }=\text { Ef. If = Ef. (b. tf3/12) }
$$

- Do: Rigidez a flexión de la lámina respecto del eje centroidal del panel:

$$
\text { Do = Ef.Ifc = Ef. [2. (b. tf). }(\mathrm{t} / 2-\mathrm{tf} / 2) 2)]
$$

- Dc: Rigidez del núcleo: Al ser el Módulo de Young a tracción de las chapas de aluminio (70.00o $\mathrm{MPa}) \mathrm{m} \mathrm{u} \mathrm{y}$ superior al del núcleo de polietileno (500 $\mathrm{MPa}$, es decir $0,7 \%)$, este término se desprecia.

E introduciendo estos parámetros en la ecuación [1] se obtiene la ecuación [2]

\begin{tabular}{|c|c|c|c|c|c|c|}
\hline Caso & $\begin{array}{l}\text { Velocidad } \\
\text { básica del } \\
\text { viento (v) } \\
\end{array}$ & $\begin{array}{c}\text { Presión dinámica } \\
q_{b}=0,5 \cdot \rho_{\text {aire }} \cdot v^{2} \\
\left(\rho_{\text {aire }}=1,25 \mathrm{~kg} / \mathrm{m}^{3}\right)\end{array}$ & $\begin{array}{l}\text { Coeficiente de } \\
\text { exposición } c_{\mathrm{e}}\end{array}$ & $\begin{array}{l}\text { Coeficiente } \\
\text { eólico } c_{p}\end{array}$ & $\begin{array}{c}\text { Presión } \\
\text { estática } \\
\mathbf{q}_{\mathrm{e}}=\mathbf{q}_{\mathrm{b}} \cdot \mathbf{c}_{\mathrm{e}} \cdot \mathbf{c}_{\mathrm{p}}\end{array}$ & $\begin{array}{l}\text { Escalón de succión } \\
\text { (§. 5.4.1. ETAG o34) }\end{array}$ \\
\hline $\begin{array}{l}\text { Torre } S^{a} \text { Cruz } \\
\text { (zona C) }\end{array}$ & $\begin{array}{l}29 \mathrm{~m} / \mathrm{s}= \\
104 \mathrm{~km} / \mathrm{h}\end{array}$ & $0,52 \mathrm{kPa}$ & 3,7 & $-1,4$ & $-2,69 \mathrm{kPa}$ & $2,80 \mathrm{kPa}$ \\
\hline $\begin{array}{l}\text { Sede de Xunta } \\
\text { (zona B) }\end{array}$ & $\begin{array}{l}27 \mathrm{~m} / \mathrm{s}= \\
97 \mathrm{~km} / \mathrm{h}\end{array}$ & $\mathrm{0}, 45 \mathrm{kPa}$ & 3,7 & $-1,4$ & $-2,33 \mathrm{kPa}$ & $2,40 \mathrm{kPa}$ \\
\hline $\begin{array}{l}\text { Viento muy } \\
\text { fuerte. AEMET } \\
\text { (valor mínimo) }\end{array}$ & $\begin{array}{c}19,7 \mathrm{~m} / \mathrm{s}= \\
71 \mathrm{~km} / \mathrm{h}\end{array}$ & $0,24 \mathrm{kPa}$ & 3,7 & $-1,4$ & $-1,24 \mathrm{kPa}$ & $1,40 \mathrm{kPa}$ \\
\hline $\begin{array}{l}\text { Viento muy } \\
\text { fuerte. AEMET } \\
\text { (valor central) }\end{array}$ & $\begin{array}{c}26,6 \mathrm{~m} / \mathrm{s}= \\
96 \mathrm{~km} / \mathrm{h}\end{array}$ & $0,44 \mathrm{kPa}$ & 3,7 & $-1,4$ & $-2,27 \mathrm{kPa}$ & $2,40 \mathrm{kPa}$ \\
\hline $\begin{array}{l}\text { Viento muy } \\
\text { fuerte. AEMET } \\
\text { (valor máximo) }\end{array}$ & $\begin{array}{l}33,3 \mathrm{~m} / \mathrm{s}= \\
120 \mathrm{~km} / \mathrm{h}\end{array}$ & $0,69 \mathrm{kPa}$ & 3,7 & $-1,4$ & $-3,57 \mathrm{kPa}$ & $3,60 \mathrm{kPa}$ \\
\hline $\begin{array}{l}\text { Límite teórico } \\
\text { del equipo de } \\
\text { ensayo (4 kPa) }\end{array}$ & $\begin{array}{l}35 \mathrm{~m} / \mathrm{s}= \\
126 \mathrm{~km} / \mathrm{h}\end{array}$ & $0,76 \mathrm{kPa}$ & 3,7 & $-1,4$ & $-3,93 \mathrm{kPa}$ & $4,00 \mathrm{kPa}$ \\
\hline
\end{tabular}

$$
\begin{gathered}
\mathrm{D}=2 \mathrm{Df}+\mathrm{Do}+\mathrm{Dc}=\mathrm{Ef} .[2(\mathrm{~b} .(\mathrm{tf}) 3 / 12)+ \\
+(2 .(\mathrm{b} . \mathrm{tf}) \cdot(\mathrm{t} / 2-\mathrm{tf} / 2) 2)]
\end{gathered}
$$

Tabla 1. Cálculos de presión estática. 


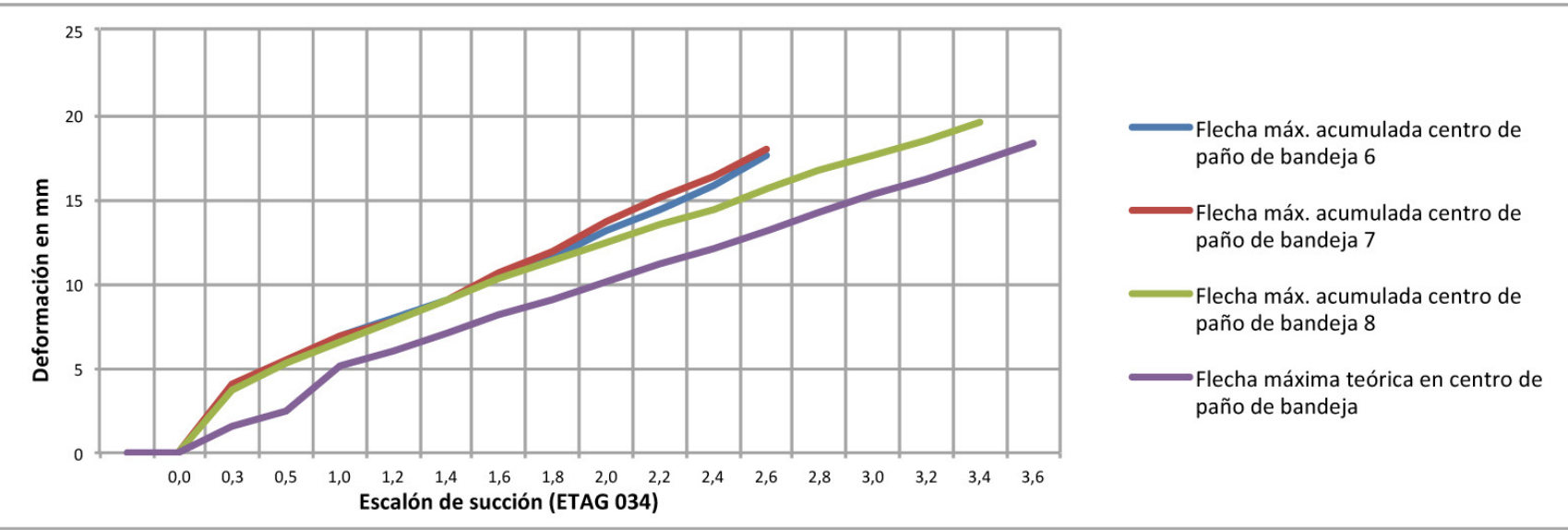

Figura 5. Deformaciones teórica y experimentales (instantánea acumulada) en centro de paño.

Las propiedades de esta placa «sólida equivalente» pueden calcularse considerando el Teorema de Steiner, igualando rigideces y expresando dicha igualdad en función de $t$ y teq, siendo t el espesor del PCA y teq el espesor de la placa sólida equivalente y Ef el módulo de Young del aluminio, resulta:

$$
\begin{gathered}
\text { Ef. }[(\text { b. tf3/12) + (b. tf3 /12) }+ \\
+ \text { (2. btf. }(\mathrm{t} / 2-\mathrm{tf} / 2) 2)]=\text { Ef. }[(\mathrm{b} . \text { teq3)/12] }
\end{gathered}
$$

Despejando teq, y considerando que $\mathrm{tf}=0,5 \mathrm{~mm}, \mathrm{yt}=4 \mathrm{~mm}$, se obtiene: teq $=[12$. $(\mathrm{t} 2-\mathrm{t}) / 4+0,0813] 1 / 3=[12(16-$ 4/4)+o,o813]1/3=3,33 $\mathrm{mm}$

3.6.2. Cálculo de cargas para alcanzar tensiones y deformaciones máximas admisibles

El caso de la bandeja tipo prevista para el ensayo de succión se modela considerando su cara vista como dos placas planas rectangulares de dimensiones $\mathrm{a}=910 \mathrm{~mm}, \mathrm{~b}=665 \mathrm{~mm} \mathrm{y}$ teq $=3,33 \mathrm{~mm}$, apoyada en tres de sus cuatro lados perimetrales, y empotrada en un lado corto b, por la presencia del rigidizador central distanciado $910 \mathrm{~mm}$ de las pestañas verticales. Se obtendrían la tensión máxima y la flecha máxima en el centro de la placa de acuerdo con las ecuaciones [4] y [5], tomadas de Roark (19), considerando la carga como uniformemente repartida. Siendo la proporción $\mathrm{a} / \mathrm{b}=1,37$, donde a es el lado mayor y b el lado menor, se obtienen los valores de los factores de forma $\alpha=0,067$ y $\beta=0,66$, interpolando respectivamente de los valores para a/b $=1(\alpha=0,030 \mathrm{y}$ $\beta=0,50)$ y para $\mathrm{a} / \mathrm{b}=1,5(\alpha=0,071 \mathrm{y} \beta=0,0067)$.

Tensión máxima (en el centro de la placa):

$$
\sigma \operatorname{adm}=(\beta . \mathrm{q} . \mathrm{b} 2 / \text { teq2 }) \leq \sigma \operatorname{adm}=51 \mathrm{MPa}
$$

Flecha máxima (en el centro de la placa):

$$
\gamma=(\alpha . \mathrm{q} . \mathrm{b} 4 / \mathrm{E} \text { teq3 }) \leq \gamma \mathrm{adm}=\mathrm{L} / 30(\mathrm{~mm})
$$

Donde:

$q$ es la presión estática negativa (succión)

$\beta$ y $\alpha$ son factores de forma, que dependen de a y b.

Siendo conocidas las dimensiones de la placa a y b, y su espesor equivalente teq $=3,3 \mathrm{~mm}$ se calcula el valor de succión en $\mathrm{kPa}$ «q» capaz de provocar un esfuerzo de tensión máxima admisible de $\sigma$ adm = $51 \mathrm{MPa}$, despejando «q» de la ecuación [4] obtenemos:

$\mathrm{q}=[(\sigma$ adm. teq2 $) /(\beta$.b2 $)] ;$ resulta introduciendo datos $=$ $1,93 \mathrm{kPa}(\approx 2,0 \mathrm{kPa})$ que sería el escalón de succión de ensayo en el que se alcanza la tensión máx. admisible a tracción de láminas de aluminio.

Y si se calcula, despejando de la ecuación [5], obtenemos el valor valor de succión para el que se alcanzaría la deformación máxima admisible L/30 = 30,33 mm, siendo $\mathrm{L}=910 \mathrm{~mm}$.

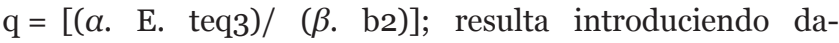
tos $=3,98 \mathrm{kPa}(\approx 4,0 \mathrm{kPa})$, que sería el valor de succión que alcanza la deformación máx. admisible en el centro de las bandejas.

\section{RESULTADOS}

En la Tabla 2 se muestran en las primeras columnas, los valores teóricos calculados de acuerdo con las indicaciones del apartado anterior (tensión de tracción de las chapas de aluminio, deformación en centro del paño de las bandejas) obtenidos para cada escalón de succión. A continuación se presentan, los valores de deformaciones o flechas acumuladas, instantáneas y diferidas, registrados por los medidores de deformación en las ubicaciones indicadas en la Figura 2.

Así, y para cada una de las bandejas ensayadas, los mayores valores deformación registrados se corresponden con donde la flexión del elemento de aplacado debía ser mayor (centros de paños de bandejas rigidizadas). Después, los valores de deformación obtenidos centros de borde horizontal inferior, y finalmente en los mínimos son aquellos registros en centros de bordes verticales.

La deformación obtenida experimentalmente es acumulada; es decir, es la resultante de sumar la deformación del aplacado, la de sus piezas de cuelgue incluyendo la de su protector de goma de 1,5 mm de espesor, y la deformación de la subestructura. Por ello, es mayor que la calculada por medios analíticos, tal y como muestra la Figura 6. Por otra parte se presentan sombreados en gris aquellos escalones de succión de viento más significativos de acuerdo con el trabajo de campo, el análisis de la peligrosidad del viento, el estudio normativo y los cálculos de cargas. 
Tabla 2. Valores de cálculo y experimentales registrados en el ensayo de succión.

\begin{tabular}{|c|c|c|c|c|c|c|c|c|c|c|}
\hline \multirow[b]{2}{*}{$\begin{array}{c}\text { Carga } \\
\text { de } \\
\text { succión } \\
(\mathrm{kPa})\end{array}$} & \multirow[b]{2}{*}{$\begin{array}{c}\sigma \\
\text { Tensión } \\
\text { tracción en } \\
\text { láminas de } \\
\text { aluminio } \\
\text { (MPa) }\end{array}$} & \multirow[b]{2}{*}{$\begin{array}{c}Y \\
\text { Flecha } \\
\text { teórica } \\
\text { centro de } \\
\text { paño } \\
\text { instantánea } \\
\text { (mm) }\end{array}$} & \multicolumn{2}{|c|}{ Bandeja 6 (PE) } & \multicolumn{3}{|c|}{ Bandeja 7 (FR) } & \multicolumn{3}{|c|}{ Bandeja 8 (FR) } \\
\hline & & & $\begin{array}{l}\text { Flecha máx. } \\
\text { acumulada } \\
\text { en } d_{1} \text { ód } \\
\text { [centro paño] } \\
\text { instantánea } \\
\text { (diferida) }\end{array}$ & $\begin{array}{c}\text { Flecha d }_{7} \\
\text { acumulada } \\
\text { instantánea } \\
\text { (diferida) }\end{array}$ & $\begin{array}{c}\text { Flecha máx. } \\
\text { acumulada } \\
\text { en } \mathrm{d}_{2} \text { ód } \\
\text { [centro paño] } \\
\text { instantánea } \\
\text { (diferida) }\end{array}$ & $\begin{array}{c}\text { Flecha d }_{9} \\
\text { acumulada } \\
\text { instantánea } \\
\text { (diferida) }\end{array}$ & $\begin{array}{c}\text { Flecha d }_{8} \\
\text { acumulada } \\
\text { instantánea } \\
\text { (diferida) }\end{array}$ & $\begin{array}{c}\text { Flecha máx. } \\
\mathrm{d}_{5} \text { ód } \\
\text { [centro paño] } \\
\text { instantánea } \\
\text { (diferida) }\end{array}$ & $\begin{array}{c}\text { Flecha d }_{11} \\
\text { acumulada } \\
\text { instantánea } \\
\text { (diferida) }\end{array}$ & $\begin{array}{c}\text { Flecha d }_{10} \\
\text { acumulada } \\
\text { instantánea } \\
\text { (diferida) }\end{array}$ \\
\hline \multirow{2}{*}{0,3} & \multirow{2}{*}{7,9} & \multirow{2}{*}{1,52} & 4,14 & 2,67 & 4,13 & 2,4 & 1,49 & 3,78 & 3,14 & 1,76 \\
\hline & & & $(0,18)$ & $(0,14)$ & $(0,13)$ & $(0,05)$ & $(0,03)$ & $(0,04)$ & $(0,05)$ & $(0,02)$ \\
\hline \multirow{2}{*}{0,5} & \multirow{2}{*}{13,18} & \multirow{2}{*}{2,53} & 5,50 & 4,57 & 5,45 & 4,46 & 2,55 & 5,36 & 4,03 & 3,14 \\
\hline & & & $(0,24)$ & $(0,19)$ & $(0,16)$ & $(0,07)$ & $(0,05)$ & $(0,14)$ & $(0,15)$ & $(0,02)$ \\
\hline \multirow{2}{*}{1,0} & \multirow{2}{*}{26,31} & \multirow{2}{*}{5,07} & 6,90 & 5,55 & 6,83 & 5,48 & 3,12 & 6,62 & 4,84 & 3,9 \\
\hline & & & $(0,38)$ & $(0,33)$ & $(0,26)$ & $(0,19)$ & $(0,13)$ & $(0,18)$ & $(0,22)$ & $(0,04)$ \\
\hline \multirow{2}{*}{1,2} & \multirow{2}{*}{31,62} & \multirow{2}{*}{6,08} & 7,95 & 6,67 & 7,84 & 6,57 & 3,71 & 7,87 & 5,70 & 4,75 \\
\hline & & & $(0,51)$ & $(0,44)$ & $(0,32)$ & $(0,24)$ & $(0,21)$ & $(0,33)$ & $(0,31)$ & $(0,19)$ \\
\hline \multirow{2}{*}{1,4} & \multirow{2}{*}{36,89} & \multirow{2}{*}{7,10} & 9,12 & 7,71 & 9,08 & 7,59 & 4,30 & 9,07 & 6,63 & 5,43 \\
\hline & & & $(0,66)$ & $(0,59)$ & $(0,46)$ & $(0,46)$ & $(0,35)$ & $(0,51)$ & $(0,44)$ & $(0,35)$ \\
\hline \multirow{2}{*}{1,6} & \multirow{2}{*}{42,16} & \multirow{2}{*}{8,11} & 10,44 & 8,72 & 10,57 & 8,75 & 5,02 & 10,23 & 7,41 & 6,32 \\
\hline & & & $(0,91)$ & $(0,74)$ & $(0,92)$ & $(0,64)$ & $(0,54)$ & $(0,64)$ & $(0,59)$ & $(0,59)$ \\
\hline \multirow{2}{*}{1,8} & \multirow{2}{*}{47,43} & \multirow{2}{*}{9,12} & 11,61 & 9,72 & 11,89 & 9,75 & 5,60 & 11,29 & 8,35 & 7,10 \\
\hline & & & $(1,18)$ & $(0,92)$ & $(1,29)$ & $(0,98)$ & $(0,48)$ & $(0,87)$ & $(0,75)$ & $(0,72)$ \\
\hline 20 & 52,7 & 10,14 & 13,16 & 11,03 & 13,65 & 11,18 & 6,51 & 12,36 & 9,20 & 7,97 \\
\hline 2,0 & & & $(1,56)$ & $(1,25)$ & $(1,83)$ & $(1,09)$ & $(1,06)$ & $(1,03)$ & $(0,92)$ & $(0,94)$ \\
\hline & & & 14,47 & 12,58 & 15,02 & 12,29 & 7,41 & 13,42 & 8,63 & 7,99 \\
\hline 2,2 & 57,97 & 11,15 & $(2,05)$ & $(1,77)$ & $(2,36)$ & $(1,13)$ & $(1,41)$ & $(0,72)$ & $(0,98)$ & $(1,19)$ \\
\hline & & & 15,77 & 14,29 & 16,27 & 13,95 & 8,26 & 14,46 & 10,97 & 9,52 \\
\hline 2,4 & 63,25 & 12,17 & $(2,35)$ & $(2,08)$ & $(2,54)$ & $(1,41)$ & $(1,64)$ & $(1,54)$ & $(1,43)$ & $(1,41)$ \\
\hline 2,6 & 68,51 & 13.18 & 17,56 & 16,02 & 17,91 & 15,42 & 9,05 & 15,61 & 12,46 & 10,70 \\
\hline 2,0 & 00,51 & 13,10 & $(2,75)$ & $(2,90)$ & $(2,92)$ & $(2,03)$ & $(2,02)$ & $(2,02)$ & $(1,91)$ & $(2,13)$ \\
\hline 28 & 7278 & 1410 & N R & $\mathrm{N} R$ & N R & N R & N R & 16,64 & 13,46 & 11,49 \\
\hline 2,0 & 73,70 & 14,19 & N.K. & N.K. & N.K. & N.K. & N.K. & $(2,25)$ & $(2,35)$ & $(2,46)$ \\
\hline 20 & 7005 & 1521 & N R & $\mathrm{N} R$ & N R & N R & N R & 17,58 & 14,91 & 12,36 \\
\hline 3,0 & 79,05 & 15,21 & N.K. & N.K. & N.K. & N.K. & N.K. & $(2,62)$ & $(2,91)$ & $(2,90)$ \\
\hline & & 1622 & & N R & N R & $\mathrm{NR}$ & N R & 18,55 & 16,31 & 13,16 \\
\hline 3,2 & 84,32 & 16,22 & N.R. & N.R. & N.R. & N.R. & N.K. & $(2,88)$ & $(3,71)$ & $(3,26)$ \\
\hline & & & N.R. & N.R. & N.R. & N.R. & N.R. & 19,59 & 18,11 & 13,99 \\
\hline 3,4 & 89,59 & 17,23 & N.K. & N.K. & N.K. & N.K. & N.K. & $(3,18)$ & $(4,95)$ & $(3,71)$ \\
\hline 3,6 & & 94,86 & & & & 然 & 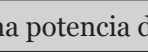 & 1 dispositiv & de ensayo. & \\
\hline
\end{tabular}

El acrónimo «N.R.» significa «no registrado», es decir que no se midieron deformaciones por la retirada de medidores de deformación LVDT. Tras superar los valores de succión 2,o kPa (el que suponía alcanzar la tensión máxima admisible a tracción de las láminas de aluminio) y $2,4 \mathrm{kPa}$ (el considerado como límite para no considerar fuerza mayor) se procedió por precaución a retirar los medidores de deformaciones de las bandejas 6 y 7, manteniéndose en la bandeja 8 .

En relación con los posibles fallos que se consideran en la Guía DITE 034 se precisa que, no se produjeron ni rotura de elementos de aplacado, ni fallos de fijación, ni desprendimientos de componentes de la subestructura pero sí se sobre- pasó la deformación permanente significativa de $3 \mathrm{~mm}$ para un valor de succión de 3,2 kPa en la bandeja 8. Finalmente, se reseña que el ensayo no pudo llegar hasta el límite teórico del equipo ( $4 \mathrm{kPa}$ ) y se detuvo en el escalón de succión de 3,6 kPa, debido a que se alcanzó su máxima potencia.

\section{CONCLUSIONES}

En el ámbito de la evaluación técnica preliminar sobre la idoneidad de empleo de estos sistemas, la resistencia frente a la succión del viento es un parámetro crítico y debe ser una de las características a determinar en primer lugar para evaluar su contribución a la seguridad de utilización del edificio. 


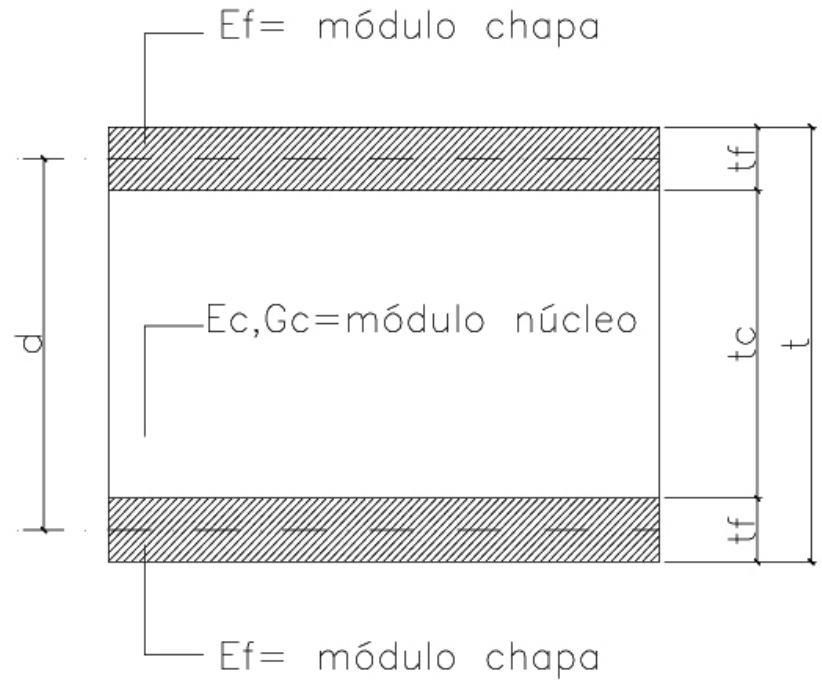

Figura 6. Deformaciones teórica y experimentales (instantánea acumulada) en centro de paño.

No se apreciaron diferencias significativas en el comportamiento de las bandejas en función de su material del núcleo ni de las configuraciones propuestas para las entalladuras (es decir aquellas de $40 \mathrm{~mm}$ de profundidad y 10,5 de lengüeta con refuerzo a base de pletina remachada frente a aquellas de $45 \mathrm{~mm}$ de profundidad y $15 \mathrm{~mm}$ de lengüeta sin refuerzo). Cualquier otra configuración diferente a la ensayada debe ser objeto de un estudio específico previo al ensayo de resistencia a la succión del viento.

Como valor umbral para una evaluación técnica favorable, se estima que en España, siempre que hayan sido adecuadamente diseñados, instalados y mantenidos, estos sistemas deben resistir una succión de viento de al menos 2,4 $\mathrm{kPa}$ sin que se produzcan los fallos de rotura descritos en la Guía DITE o34, esto es, ni rotura de elementos de aplacado, ni fallos de fijación, ni desprendimientos de componentes de la subestructura.
Asimismo, para una evaluación técnica favorable deben limitarse las deformaciones permanentes y residuales, del aplacado y no sólo en el centro de la cara vista de la bandeja. También se tendrá en cuenta el centro de la pestaña horizontal inferior.

En este último caso, puede alcanzarse valores de deformación residual superiores a los del centro de la cara vista si las lengüetas de las entalladuras inferiores de la bandeja se deformaran más, es decir, se abrieran más, que las de las entalladuras superiores. Esto supondría un cambio de modelo mecánico de comportamiento de la cara vista. Así para una bandeja sin rigidizadores adheridos, en vez de una placa apoyada en su perímetro, habría que considerar el modelo de placa apoyada en tres lados y uno, el horizontal inferior, como libre o en voladizo.

El criterio establecido en el Estado del Arte de limitar la deformación instantánea a L/30, siendo L la distancia entre pestañas verticales o entre pestaña y rigidizador vertical adherido se cumple sobradamente si las láminas de aluminio presentan límites elásticos Rpo,2 por encima del valor mínimo establecido en la correspondiente norma.

El criterio establecido en el Estado del Arte de limitar la deformación residual o permanente y significativa a un máximo de $3 \mathrm{~mm}$, por su apreciación visual, debe sustituirse por otro criterio definido, al igual que para las deformaciones instantáneas, en función de la distancia L indicada anteriormente. Para una estimación preliminar puede considerarse un valor de $\mathrm{L} / 300$.

Como futuras líneas de investigación se propone la incorporación del cálculo por el método de elementos finitos a la evaluación técnica preliminar de la resistencia frente a la succión de este tipo de sistemas, partiendo de un proceso previo de comparación entre simulaciones por ordenador y resultados experimentales de laboratorio, en particular de los efectos de las rachas de viento.

\section{REFERENCIAS}

(1) Blázquez Morales, A. (2014). Metodología para la evaluación de productos innovadores de construcción (Preassessment/Preevaluación Dit). Tesis doctoral. Madrid: Universidad Politécnica de Madrid.

(2) Blachére, G. (1978). Saber construir. Editores Técnicos Asociados. ISBN: 84-7146-032-7.

(3) ASEFAVE. Asociación Española de Fabricantes de Fachadas Ligeras y Ventanas S.L. Manual de Producto. Fachadas Ligeras. Ed. Tecnopress Ediciones. ISBN-10: 8493902314.

(4) Lahoz, E. (2008). Documento de Idoneidad Técnica DIT 405R/o8. Sistemas de revestimiento de fachadas ventiladas mediante bandejas o placas procedentes de paneles compuestos de aluminio. Instituto de Ciencias de Construcción Eduardo Torroja (IETcc-CSIC).

(5) Lahoz, E. (2012). Documento de Idoneidad Técnica DIT 553A/12. Sistemas de revestimiento de fachadas ventiladas mediante bandejas o placas procedentes de paneles compuestos de aluminio. Instituto de Ciencias de Construcción Eduardo Torroja (IETcc-CSIC).

(6) AENOR. (2000). UNE 41957:200o Anclajes para revestimientos de fachadas de edificios. Parte 1: Subsistemas para revestimientos ligero. Asociación Española de Normalización (AENOR).

(7) Martín León, F., et al. Estudio de la tormenta tropical Delta. AEMET. http://www.aemet.es/es/conocermas/recursos_en_linea/publicaciones_y_estudios/estudios/detalles/Estudio_de_la_tormenta_tropical_Delta.

(8) AEMET. (2014). http://www.aemet.es/documentos/es/serviciosclimaticos/vigilancia_clima/resumenes_climat/ccaa/ galicia/avance_climat_gal_feb_2014.pdf.

(9) AEMET. (2015). Capítulo II. Definición de las variables meteorológicas, apdo. F. Manual de uso de términos meteorológicos, pp. 31-33. Agencia Estatal de Meteorología. Ministerio de Agricultura, Alimentación y Medio Ambiente. Catálogo de Publicaciones de la Administración General del Estado. Depósito Legal: M-32801-2014.

(10) Ministerio de Agricultura, Alimentación y Medio Ambiente, AEMET. (2013). Plan nacional de predicción y vigilancia de fenómenos meteorológicos adversos meteoalerta. Ed. Versión: 5. Fecha: 9-7-2013. http://www.lamoncloa.gob.es/ espana/eh15/medioambiente/Documents/Fen\%C3\%B3menos\%2ometeorol\%C3\%B3gicos.pdf. 
(11) Ministerio de Economía. (2004). Real Decreto 300/2004, de 20 de febrero, por el que se aprueba el Reglamento del seguro de riesgos extraordinarios. Boletín Oficial del Estado, 47.

(12) Ministerio de Economía y Hacienda. (2011). Real Decreto 1386/2011, de 14 de octubre, por el que se modifica el Reglamento del seguro de riesgos extraordinarios. Boletín Oficial del Estado, 259.

(13) Tomás, A., Morales, M. (julio-septiembre 2012). Revisión y estudio comparativo de la acción del viento sobre las estructuras empleando Eurocódigo y Código Técnico de la Edificación. Informes de la Construcción, 64(527): 381-390. ISSN: 0020-0883.

(14) AENOR. (2007-2010). UNE EN 1991-1-4:2007 + UNE EN 1991-1-4/AC: 2010 + UNE EN 1991-1-4/A1: 2010. Eurocódigo 1. Acciones en estructuras. Parte 1-4. Acciones generales. La acción del viento. Asociación Española de Normalización (AENOR).

(15) Pindado Carrión, S., Franchini, S., Meseguer Ruiz, J. (2007). Aerodinámica civil y energía eólica, Anexo B: Cargas en cubiertas debidas a torbellinos cónicos. IDR-UPM.

(16) Allen, H. G. (1969). Analysis and design of structural sandwich panels. Oxford: Pergamon Press.

(17) Plantema, F. J. (1966). Sandwich Construction. John Wiley \& Sons.

(18) Zenkert, D. An introduction to Sandwich Construction. Engineering Materials Advisory Services LTD. ISBN: 094781778.

(19) oung, W. C., Budynas, R. G. (2002). Chapter 11. Flat Plates. Roark's Formulas for Stress and Strain, pp. 508 y 522.7 th ed. McGraw-Hill. ISBN: 0-07-072542-X. 